\title{
AN OPEN TRIAL OF INTEGRATIVE THERAPY FOR GENERALIZED ANXIETY DISORDER
}

\author{
MICHELLE G. NEWMAN, LOUIS G. CASTONGUAY, \\ THOMAS D. BORKOVEC, AARON J. FISHER, AND SAMUEL S. NORDBERG \\ The Pennsylvania State University
}

Cognitive-behavioral therapy (CBT), although effective, has the lowest average effect size for generalized anxiety disorder (GAD), when compared to effect sizes of CBT for other anxiety disorders. Additional basic and applied research suggests that although interpersonal processes and emotional avoidance may be maintaining GAD symptomatology, CBT has not suffciently addressed interpersonal issues or emotion avoidance. This study aimed to test the feasibility and preliminary efficacy of an integrative psychotherapy, combining CBT with techniques to address interpersonal problems and emotional avoidance. Eighteen participants received 14 sessions of CBT plus interpersonal emotional processing therapy and three participants (for training and feasibility purposes) received 14 sessions of CBT plus supportive listening. Results showed that the integrative therapy significantly decreased GAD symptomatology, with maintenance of gains up to 1 year following treatment. In addition, compari-

Michelle G. Newman, Louis G. Castonguay, Thomas D. Borkovec, Aaron J. Fisher, and Samuel S. Nordberg, Department of Psychology, The Pennsylvania State University.

This research was supported in part by National Institute of Mental Health Research Grant MH-58593. We thank Jeremy Safran for his role in the training of therapists and monitoring of therapist competence.

Correspondence regarding this article should be addressed to Michelle G. Newman, Department of Psychology, The Pennsylvania State University, University Park, PA 16802. E-mail: mgn1@psu.edu sons with extant literature suggested that the effect size for this new GAD treatment was higher than the average effect size of CBT for GAD. Results also showed clinically significant change in GAD symptomatology and interpersonal problems with continued gains during the 1-year follow-up. Implications of these results are discussed.

Keywords: integrative therapy, cognitive-behavioral therapy, generalized anxiety disorder

Cognitive-behavioral therapy (CBT) for generalized anxiety disorder (GAD) has been found to produce significant improvement, which is maintained for up to 1 year following treatment termination. Studies also show that CBT generates greater GAD improvement than no treatment, analytic psychotherapy, pill placebo, nondirective therapy, and placebo therapy (Borkovec \& Ruscio, 2001). Despite its general efficacy, however, studies also show that CBT is not beneficial for all clients. Furthermore, CBT leads to a smaller percentage of high endstate functioning in GAD than in other anxiety disorders (Brown, Barlow, \& Liebowitz, 1994).

One hypothesis for the limitation of its impact is that CBT protocols for GAD have not included techniques to address important factors associated with the maintenance of this disorder, such as interpersonal problems and emotional avoidance. In terms of interpersonal difficulties, research shows that worry content for those with GAD is more frequently about interpersonal concerns than any other topic (Breitholtz, Johansson, \& Öst, 1995; Roemer, Molina, \& Borkovec, 1997), that worry correlates more highly with social fears than nonsocial fears (Borkovec, Robinson, Pruzinsky, \& 
DePree, 1983), and that social phobia is the most frequent comorbid anxiety disorder to GAD (Borkovec, Abel, \& Newman, 1995; BrawmanMintzer et al., 1993; Newman, Przeworski, Fisher, \& Borkovec, 2007). Compared to people without GAD, those with GAD also view their relationships with elevated hypervigilence and suspiciousness (Gasperini, Battaglia, Diaferia, \& Bellodi, 1990), and are more likely to over- or underestimate the extent of their negative impact on others (Erickson \& Newman, 2007). Moreover, Pincus and Borkovec (1994) found that compared to those without GAD, persons with GAD had significantly more interpersonal distress and interpersonal rigidity across different situations. They also found that mean scores of GAD individuals on five of eight Inventory of Interpersonal Problems Circumplex Scales (IIP-C; Alden, Wiggins, \& Pincus, 1990) were significantly higher than psychiatric norms.

Data also suggest problems with intimate relationships (Newman, 2000). For example, in a sample of 4,933 married couples, marital discord was independently and more strongly associated with GAD than major depression, mania, dysthymia, social phobia, simple phobia, agoraphobia, panic, and alcohol dependence after controlling for demographic variables, comorbid disorders, and quality of other relationships (Whisman, Sheldon, \& Goering, 2000). The latter study also found that GAD predicted a lack of close friendships. Additional studies show that parents with GAD had significantly higher rates of dysfunctional relationships with their spouses and children compared to parents without GAD (Ben-Noun, 1998).

Given that people with GAD worry predominantly about their relationships, their objective difficulty sustaining healthy relationships is likely to reinforce and maintain these worries. Taken together, these findings suggest that relationship difficulties may contribute to the maintenance of GAD. As such, it is possible that changes in relationship difficulties may improve GAD treatment outcome.

Similar to the evidence for interpersonal difficulties, there is evidence that people with GAD are uncomfortable with and avoidant of emotions (Borkovec \& Newman, 1998). In both clinical and nonclinical GAD samples, worry and GAD were associated with a propensity to try to avoid or control internal experiences, as well as a fear of losing control over emotional responses (Turk,
Heimberg, Luterek, Mennin, \& Fresco, 2005). In addition, in GAD analogues, both anxious and sad mood inductions led to heightened reports of anxiety and greater difficulty regulating negative mood compared to controls (Llera \& Newman, 2007; Mennin, Heimberg, Turk, \& Fresco, 2005). Further, GAD analogues showed a defensive response even to positive emotional stimuli (Yamasaki, Behar, \& Ray, 2002) and were objectively rated as demonstrating more anxiety and sadness than control participants, in response to sad and conflictual emotional disclosures by a confederate (Erickson \& Newman, 2007).

There is also a large body of empirical literature suggesting that worry, the cardinal feature of GAD, serves as a strategy to avoid emotional experience. For example, individuals with GAD report that worry helps to distract them from more emotional issues (Borkovec \& Roemer, 1995). There is also evidence that verbal linguistic thought is used spontaneously by people to avoid emotional arousal associated with emotionally evocative stimulus materials (Tucker \& Newman, 1981). These findings are consistent with several other studies (Borkovec \& Hu, 1990; Llera \& Newman, 2007) suggesting that the verbal linguistic nature of worry enables avoidance of emotion by reducing imagery and concomitant physiological responses. In line with Foa and Kozak's (1986) emotional processing theory, such a lowered response to feared stimuli means that the full fear structure will not be accessed. Without full emotional processing, fear is maintained.

Despite the above evidence indicating that both interpersonal issues and emotional avoidance may be highly relevant to the maintenance of GAD symptoms, current CBT protocols may not be adequate to successfully address these issues in GAD. Borkovec, Newman, Pincus, \& Lytle (2002) found that CBT for GAD failed to make a significant change in 6 of 8 IIP-C scales at post-therapy, and most clients continued to score at least one standard deviation $(S D)$ above normative levels on at least one IIP-C subscale, This study also found that pretherapy interpersonal problems (dominant/hostile, intrusive/needy, vindictive/selfcentered) predicted negative CBT outcome. Moreover, interpersonal problems not successfully treated by CBT at postassessment were predictive of failure to maintain follow-up gains. Such evidence points to the necessity of including therapy techniques to specifically address patterns of interpersonal problems, including the client's 
contribution to maintaining maladaptive ways of relating with others.

Similar to its failure to address interpersonal problems, CBT for GAD has failed to include interventions to target emotional avoidance and discomfort (Newman, Castonguay, Borkovec, \& Molnar, 2004). In a study by Borkovec and Costello (1993), the level of emotional processing was found to be significantly lower in CBT than in a reflective listening condition. This finding is consistent with process research literature suggesting that "CBT attempts to control or reduce patient's feelings" (Blagys \& Hilsenroth, 2000, p. 172). Studies have also found that higher levels of emotional experiencing were associated with a positive outcome in CBT (e.g., Castonguay, Goldfried, Wiser, Raue, \& Hayes, 1996). Taken together, these basic and applied findings suggest that adding techniques specifically designed to help GAD clients deeply experience and process uncomfortable emotions may help them to reduce their chronic worrying. Within a CBT framework, such an intervention can be viewed as a means for exposure to feared stimuli (i.e., feared emotions), and within an interpersonal framework, emotional deepening can be viewed as a means for identifying interpersonal needs.

The first goal of this preliminary open trial was to determine whether it was feasible to train therapists in a well-controlled protocol therapy for GAD that added interpersonal and emotional processing interventions (I/EP) to cognitivebehavioral therapy (CBT). As detailed elsewhere (Newman et al, 2004), the conceptual basis for addition of these components was derived, in large part, from Safran and Segal's expansion of cognitive therapy (Safran, \& Segal, 1990). Second, we were interested in examining (albeit tentatively) whether this integrative treatment showed promising efficacy.

\section{Method}

Rather than involving a seamless integration of CBT and I/EP techniques (where any of these techniques could be used at any time), our design, for scientific reasons, involved a separation and sequential combination of two distinct therapeutic segments (i.e., 50-min of CBT, followed by 50-min I/EP). This preliminary study was planned to allow us to conduct a later randomized trial using an additive design, argued to be one of the most powerful designs available for therapy outcome research in its efforts to identify specific causal ingredients (For a full description of the advantages of additive designs over other research designs in terms of internal validity see Behar \& Borkovec, 2003). In such an eventual investigation, the integrative combination therapy examined in the current open trial would be compared to a 50-min CBT, followed by a 50-min supportive (SL) condition. Controlling for common factors, such a between-group additive design is not only a method to directly answer whether CBT can be improved upon, but it is also the only scientifically available method to unambiguously examine whether I/EP causes a significant increment in efficacy beyond CBT. Without their separation, the amount of time spent in the various segments would be more difficult to determine, and thus the conclusions about the specific contributions of the two segments would be more ambiguous. In as much as the current study was planned as a preliminary step for the randomized trial mentioned above, we also tested the feasibility of the control condition (CBT + SL) with three participants (one per therapist involved in the current open trial).

\section{Participants}

Admission criteria included agreement between two diagnostic interviewers on: a principal diagnosis of DSM-IV GAD, an Assessor Severity of GAD rating of 4 (moderate) or greater, absence of concurrent psychosocial therapy, no medical contributions to the anxiety, and absence of substance abuse, psychosis, and organic brain syndrome. Of 50 people who contacted our project, 14 were ruled out by phone screening; diagnostic interviews ruled out an additional 12 for not meeting admission criteria. Of 24 admitted clients, three dropped out at early stages of treatment $(0$ in CBT $+S D$ and 3 in CBT + I/EP; nonsignificant by chi-square analysis). No clients were removed for deteriorating conditions during therapy. The 21 clients who completed treatment averaged 37.89 years of age $(S D=11.03)$, and duration of the GAD problem averaged 12.81 years $(S D=10.86)$. Sixteen of the clients were female $(76.2 \%)$, and five $(23.8 \%)$ were male. Ethnicity was represented by 19 Caucasian, 1 Asian American, 1 Hispanic, and 1 Middle Eastern client. Axis I comorbidity was $47.6 \%(n=$ 10) social phobia, $47.6 \%(n=10)$ simple phobia, $23.8 \%(n=5)$ dysthymia, $9.5 \%(n=2)$ major 
depression, $9.5 \%(n=2)$ post-traumatic stress disorder, $4.8 \%(n=1)$ panic disorder, and $4.8 \%$ $(n=1)$ agoraphobia. Axis II comorbidity was $38.1 \%(n=8)$ obsessive-compulsive, $28.6 \%$ $(n=6)$, avoidant, $28.6 \%(n=6)$ depressive, $4.8 \%(n=1)$ borderline, $4.8 \%(n=1)$ paranoid, and $4.8 \%(n=1) 4.8 \%(n=1)$ schizotypal personality disorder. Mental health practitioners had referred two of the clients; the remainder had responded to media advertisements. Four clients were taking psychotropic drugs for anxiety; they agreed to maintain dosage and frequency during therapy with their physician's approval, and daily diary monitoring of drug use indicated compliance with this request.

Three therapists, all Ph.D. psychologists, conducted the therapy. Two of the therapists had primary orientations of psychodynamic therapy and one was cognitive-behavioral. Assignment to therapist was random within restraints of availability and caseload. For the initial 6-months of the project (7/96-12/96), therapists were formally trained by reading treatment manuals, listening to tapes, discussion, role-playing, and engaging in a 5-day intensive training experience with an expert in both CBT and I/EP interventions. This was followed by the treatment of one pilot client for each therapist with joint supervision by the first three authors, and all three therapists. The first and third author provided weekly individual supervision of each therapist throughout the project for the I/EP and CBT segments, respectively.

\section{Procedure}

Selection and assessor outcome ratings. Clients were enrolled over a 2-year period. Advanced clinical graduate students trained in diagnostic interviewing conducted a 30-min phone interview to determine diagnostic suitability. They then administered in person the Anxiety Disorders Interview Schedule-IV(ADIS-IV; Brown, Di Nardo, \& Barlow, 1994), the Hamilton Anxiety Rating Scale (HARS; Hamilton, 1959), the Structured Clinical Interview for $D S M-I V$ Axis II Personality Disorders (First, Spitzer, Gibbon, Williams, \& Benjamin, 1994), as well as the Assessor Severity of GAD Anxiety Symptoms ( $0-8$ point scale). Because GAD is characterized by the lowest degree of interrater reliability among the anxiety disorders (Brown, Campbell, Lehman, Grisham, \& Mancill, 2001), a second ADIS-IV, to reduce the likelihood of false positive cases, was given within 2 weeks by the therapist who would see the client in therapy upon acceptance into the trial. Each of the three therapists ruled out one client at this point and inter-rater agreement was $91 \%$. A random subsample of $20 \%$ of pretreatment audiotapes of interviews conducted by the primary assessors from our last therapy trial revealed excellent agreement on assessor severity ratings of the primary and comorbid disorders with correlations ranging from .93-1. A briefer version of the ADIS (assessing only those diagnoses identified at pretherapy) and the Assessor Severity rating scale were readministered 10-14 days after the last therapy session and at 6-months follow-up assessment; the complete ADIS and rating scales were given at 12-months follow-up. The same assessor administering the preassessment to a client also administered the postassessment to that client at follow-up whenever possible. Assessors were uninformed of therapy condition.

At a separate questionnaire session, clients completed the State-Trait Anxiety Inventory-Trait (STAI; Spielberger, Gorsuch, Lushene, Vagg, \& Jacobs, 1983); the Reactions to Relaxation and Arousal Questionnaire (RRAQ; Heide \& Borkovec, 1983); and the Penn State Worry Questionnaire (PSWQ; Meyer, Miller, Metzger, \& Borkovec, 1990). These measures were given again at the postassessment and follow-up ADIS assessments.

\section{Self-Report Outcome Measures}

Client daily diary. This is a measure of chronic anxiety and worry (Borkovec \& Costello, 1993). Four times a day (upon arising, end of morning, end of afternoon, and end of evening) clients rated their average level of anxiety during the preceding time block on a 100-point scale. This measure was completed during the 2 weeks before therapy, during therapy (including the 10-14 days after Session 14 for postassessment), and for 1 week before each follow-up. Two-week retest reliability was .8 in our previous therapy trial and convergent and discriminant validity was demonstrated. Average compliance with this measure in the current trial was $84 \%$.

STAI-T. This 20-item scale is used to measure trait anxiety. Internal consistency reliability was shown to be high (in the .80's and .90's) and retest reliability was much higher for the trait form (high 70's) than the state form (from .27 to .54). Convergent and discriminant validity has also been demonstrated for this questionnaire (Spielberger et al., 1983). 
HARS. This clinician-administered scale was developed to assess anxious symptoms among already anxious individuals (Hamilton, 1959). Internal consistency estimates $(\alpha=.92)$ are excellent (Kobak, Reynolds, \& Greist, 1993), interrater reliability ranges from an intraclass corelation coefficient (ICC) of .74-.96 (Bruss, Gruenberg, Goldstein, \& Barber, 1994) and retest reliability was .86 across 2 days. In addition, this measure demonstrated good convergent (Snaith, Harrop, Newby, \& Teale, 1986), and discriminant validity (Kobak et al., 1993), and sensitivity to treatment change (Maier, Buller, Philipp, \& Heuser, 1988).

$R R A Q$. This is a 9-item, factor-analytically derived measure of fear of relaxation (Heide \& Borkovec, 1983). Internal consistency $(\alpha=.85)$ convergent, discriminant, and retest reliability $(r=$ .83 ) of this scale over a 2-week period were demonstrated (Newman et al., 2002). This measure has also demonstrated sensitivity to change associated with psychotherapy for GAD (Borkovec \& Inz, 1990).

$P S W Q$. This 16-item scale measures the frequency and intensity of worry (Meyer et al., 1990). Internal consistency $(\alpha=.91)$, sensitivity to change from psychotherapy, convergent, discriminant, and retest reliability (ranging from .74-.93) across 2-10 weeks for this scale have been demonstrated (Brown, Antony, \& Barlow, 1992; Meyer et al., 1990; Molina \& Borkovec, 1994).

Secondary outcome measures. The Inventory of Interpersonal Problems Circumplex (IIPC Alden et al., 1990) was administered at all assessment periods to assess the impact of treatment on aspects of functioning that were not part of the GAD symptoms, but were the focus of intervention in the I/EP segment. The IIP assesses eight scales that form a circumplex of interpersonal problems around the dimensions of dominance and nurturance. The scales show strong convergence between self- and peer-rating profiles, that discriminate subgroups of depressed clients possess homologous structure with dispositional interpersonal variables, and good alpha (.72-.85) coefficients (Alden \& Phillips, 1990; Bartholomew \& Harowitz, 1991).

\section{Therapy}

CBT. All clients received CBT during the first $50 \mathrm{~min}$ of each of the 2-hr Sessions 1-14 (each of the two 50-min segments of each session was followed by a 10-min period to complete process measures, which will be the focus of future publications). These techniques targeted intrapersonal aspects of anxious experience and included the following methods from the most comprehensive CBT protocol previously developed and tested at Penn State University (Borkovec et al., 2002): (a) CBT model, and rationale; training in selfmonitoring of environmental, somatic, active, imaginal, and thought (especially worry) cues that trigger anxiety spirals with special emphasis on increasingly early cue detection; external and especially internal cue hierarchy development; formal progressive relaxation (modified over sessions from 16 muscle groups, 4 muscle groups, 4 group-recall, and counting (Bernstein \& Borkovec, 1973); training in cue-controlled and differential relaxation; applied relaxation training (AR); development of coping self-statements to use in response to cues; and employment of selfstatements and AR during formal self control desensitization (SCD) imagery for rehearsal of coping responses. Hierarchies for SCD were constructed from pretherapy questionnaires and ADIS information, daily self-monitoring, and insession discussion with the client. (b) Cognitive therapy (based on Beck, Emery \& Greenberg, 1985) involved presentation of the role of cognition in anxiety; training in self-monitoring of early worry and automatic thought occurrence; identification of cognitive predictions, interpretations, beliefs, and assumptions underlying the threatening nature of events or cues; logical analysis; examination of evidence supporting automatic thoughts; labeling of logical errors; decatastrophization; generation of alternative thoughts and beliefs; early application of these alternatives to daily living; creation of behavioral experiments to obtain evidence for new beliefs; and use of cognitive perspective shifts learned in cognitive therapy during SCD rehearsals.

Interpersonal/emotional processing segment. This segment was informed by Safran and Segal's (1990) model of interpersonal schema which provides a comprehensive and coherent integration of cognitive, interpersonal, and emotional issues in human functioning and therapy change. However, in contrast to Safran's model, for the purpose of tailoring the treatment to GAD, this segment was specifically designed to address interpersonal problems and to facilitate emotional deepening and acceptance, without direct integration of cognitive techniques. Based on data on the 
nature (verbal linguistic) and function (emotional avoidance) of worry described above, we assumed that the examination and challenge of worry as used in our previous CBT trials would interfere or hinder the fostering emotional processing at the core of I/EP. As described above, the decision not to directly integrate these cognitive techniques in this therapy segment was also based on the scientific importance of separating the CBT and I/EP segments.

CBT always preceded I/EP because engaging in alliance rupture repair methods (an interpersonal intervention) was allowed only in the I/EP segments. Thus, if a rupture occurred during CBT, it could be repaired during the next hour. If CBT had followed I/EP in each session, a full week would transpire before repair techniques could be employed. This would be potentially detrimental to the client and to the effectiveness of the therapy.

Patients were told that current interpersonal difficulties and failure to access primary emotions are involved in the generation of anxiety and worry. Consequently, the goals of this portion of therapy are: (a) identification of interpersonal needs, past and current patterns of interpersonal behavior that attempt to satisfy those needs, and emotional experience that underlies all of these, and (b) generation of more effective interpersonal behavior to better satisfy the needs. Therapy made use of four primary and interrelated domains to accomplish these goals: (a) current problems in interpersonal relationships, including negative impact clients have on others, (b) interpersonal developmental origins (e.g., attachment and trauma experiences) of relationship difficulties, (c) interpersonal patterns and problems (including ruptures in the therapeutic alliance) that emerged in the relationship with the therapist, and (d) emotional processing in the here-and-now of affects associated with these domains. Focus on these four domains was guided by eight principles, including emphasis on phenomenological experience; therapists' use of their emotional experience to identify interpersonal markers; use of the therapeutic relationship to explore affective processes and interpersonal patterns, with therapists' assuming responsibility for their role in the interactions; promotion of generalization via exploration of between-session events and provision of homework experiments; detection of alliance ruptures and provision of emotionally corrective experience in their resolution; processing of patient's affective experienc- ing in relation to past, current, and in-session interpersonal relationships; and use of skill training methods (e.g., assertion, problem-solving, communication training, role-playing) to provide more effective interpersonal behaviors to satisfy needs. In I/EP, therapists explicitly identified disaffiliative emotions, attended to their own emotional reactions to patients, and attempted to encourage patients to openly communicate their feelings with a goal of repairing any disaffiliation.

$S L$ segment. This segment, used with only three clients for training purposes, was adopted directly from the SL manual of our prior trials (see Borkovec et al., 2002). Clients were told that this segment involves exploration of important life experiences in a quiet, relaxed atmosphere where the therapist's goals are to facilitate and deepen knowledge about self and anxiety. This portion of therapy was presented as an inward journey that might be additionally helpful in changing anxious experience and increasing selfconfidence. The therapist's role was to provide an opportunity for self-reflection in a safe environment to facilitate change. The clients' role was to emphasize their unique efforts to discover new strengths through introspection. The manual instructed therapists to create an accepting, nonjudgmental, empathic environment, and to facilitate the allowing and accepting of ongoing experience via supportive statements, reflective listening, and empathic communications. The therapist was not allowed, however, to use any methods either to deepen the emotional experience of the client or to facilitate client recognition or accessing of more primary affects. Any other methods, including any direct suggestions, advice, or coping methods, were prohibited during this portion of the session.

Adherence checks. Trained clinical graduate students not involved in a client's ADIS or questionnaire sessions rated every therapist utterance on $100 \%$ of three audio-taped sessions randomly selected from early, middle, and late sessions for each client. Ratings were categorical by content of therapists' verbalizations and classified by checklist entries for all intervention types allowed and not allowed by each protocol. Ratings were separately obtained from the CBT, I/EP, and SL segments of each chosen session. Of the 5,163 checked utterances, only three were coded as minor protocol breaks, all three occurring in I/EP segments. In one of these occurrences, the therapist focused on how the client's worry 
prevented him from enjoying himself. The other two "nonallowed" utterances referred back to the same incident, where the therapist referring back to the CBT segment instructed the client to use SCD outside the session. No I/EP Interventions were coded in CBT sessions.

Quality checks. Dr. Safran rated $100 \%$ of two randomly selected sessions (1st and 2 nd half of sessions) for each client, using (a) our Cognitive Therapy Quality Scale, incorporating Young and Beck's (1980) Scale into our furtherelaborated version to assess the quality of the $1 \mathrm{st}$ 50-min CBT intervention, and (b) our I/EP and SL Scales, developed for this project from the manual sections for these segments and on items contained in other competency scales (e.g., Safran \& Segal, 1990) to assess the quality of the 2nd 50-min intervention (I/EP or SL). All rated sessions met the a priori criteria (a score of 3 ("satisfactory") or greater in the CBT and I/EP segments on an overall rating item $(0=$ poor; 6 = excellent) of the therapist for this session; an average of 3 or above (based on the same likert scale) on the 4 items of the SL reflective listening quality scale [which did not have an "overall" quality rating]) for the client to be included in the final analyses. Average ratings were 4.71 for CBT, 4.25 for I/EP, and 5.0 for SL.

\section{Results}

\section{Pre-Post and Post-Follow-Up Changes}

We first examined therapeutic progress from pre- to posttreatment and from posttreatment to follow-up assessment. Because these repeated measurements at successive times came from the same individual, we elected to use an analytic strategy that takes into account the nonindependence of data points. Linear mixed-effects models sometimes referred to as hierarchical linear modeling (HLM), multilevel modeling, or random-effects models account for such correlation by incorporating it into the model. Withinindividual variation, referred to as random effects, can be modeled separately from systematic sources of variation in the dependent variable, known as fixed effects. Random effects are variance components that ultimately indicate whether, in this case individuals, differed significantly from the mean. Fixed effects are the actual regression estimates for the model. Mixed effect models also have the ability to accommo- date missing data points. Missing cases are ignored only within a given missing time point, allowing the individual to be retained within the analysis. SL individuals were not in these analyses given the small sample size, which limited power to find existent differences as well as limiting the generalizability of any such findings.

Random effects were determined with restricted maximum likelihood estimation, a more conservative estimation method. Restricted maximum likelihood is only able to test the significance of variance components and so cannot be used to test the fixed effect components of a given model. For this reason, we investigated possible significant random effects before estimating the significance of our fixed effects. Random effects for individual variation at the intercept were first entered into the models for each of the six dependent variables and found to be significant. Random effects for individual variation in slope for time were then entered into each model, were not found to be significant, and thus were removed from the models. Fixed effects were then estimated using full maximum likelihood. All models were constructed using a piecewise analysis of time as opposed to a single time coefficient.

Piecewise analyses allow for the representation of discrete multiple time periods by modeling separate variables (and therefore separate coefficients and slopes) for these periods. In the case of a treatment outcome study such as the current study, the treatment and follow-up periods can be conceptualized as discrete and yet are represented within the same model. Piece1 was therefore conceptualized as the treatment period, from baseline to endof-treatment. Piece 2 was conceptualized as the posttreatment period including 6- and 12-month follow-ups. Modeling of time in a piecewise fashion significantly improved fit across all analyses. ${ }^{1}$

Piecewise analyses for all six dependent variables revealed a significant negative slope for Piece1 and a nonsignificant slope for Piece 2. Table 1 reports the $\beta$-weights for these analyses. These results indicated a strong improvement in clients across measures from pre- to posttreatment and maintenance of those gains during the

\footnotetext{
${ }^{1}$ Prior research has examined individual trajectories during treatment period and follow-up. However, given that we did not find significant between-subject variation in linear, quadratic, or cubic slope (i.e. random effects), such an examination was not warranted in the current study.
} 
TABLE 1. Beta Weights Representing Change from Pre- To PostTreatment (Piece 1) and PostTreatment to 1 Year Follow-Up (Piece 2)

\begin{tabular}{lccc}
\hline \hline & $\begin{array}{c}\text { Pretreatment to } \\
\text { posttreatment } \\
\beta \text {-weight }\end{array}$ & $\begin{array}{c}\text { Posttreatment } \\
\text { to 1 year FU } \\
\beta \text {-weight }\end{array}$ & $\begin{array}{c}\text { Piece } 1 \times \text { Baseline* } \\
\beta \text {-weight }\end{array}$ \\
\hline Assessor severity & $-0.87^{+++}$ & .004 & $-.20^{+}$ \\
Daily diary & $-3.13^{+++}$ & -.003 & $-.14^{+++}$ \\
PSWQ & $-4.86^{+++}$ & .20 & $-.18^{+}$ \\
HARS & $-3.44^{+++}$ & -.06 & $-.17^{+++}$ \\
STAI-T & $-3.18^{+++}$ & .16 & $-.17^{++}$ \\
RRAQ & $-2.05^{+++}$ & .12 & $-.11^{++}$ \\
\hline
\end{tabular}

Note. $\quad$ PSWQ $=$ Pennsylvania State Worry Questionnaire, HARS = Hamilton Anxiety Rating Scale, STAI-T = State Trait Anxiety Inventory - Trait Version; RRAQ = Response to Relaxation and Arousal Questionnaire.

$+p<.05, \quad++p<.01, \quad+++p<.001$.

* Separate models were constructed to determine piecewise slopes and piece $1 \times$ baseline interactions.

follow-up period. For instance, analyses of assessor severity revealed a -.87 slope from pre- to posttreatment which, given the 4-month treatment period, resulted in a mean decrease of 3.48. The nonsignificant slope for Piece 2 demonstrates the maintenance of these gains over the 1-year follow-up period (see Figure 1).

Whereas the significant change found for Piece 1 suggests a linear improvement during treatment, the limited number of measurement occasions used in these analyses (pre- and post- treatment) precludes the testing of nonlinear (polynomial) trends. Articulation of polynomial trends requires $n+1$ time points for a given polynomial. For instance, in order to demonstrate a quadratic trend, three time points are required, a cubic trend, four time points and so forth. In order to examine possible higher-order trends within the treatment period, diary data collected at each session were analyzed. When such anal-

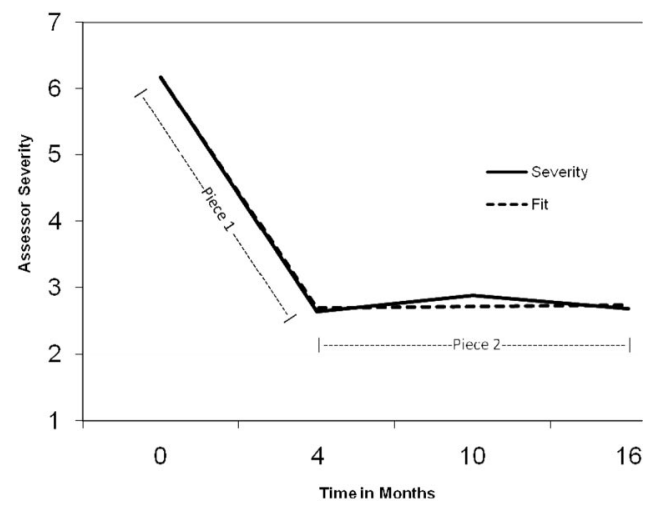

FIGURE 1. Actual and Modeled (Fit) Assessor Severity over Time. yses were conducted, the best-fit model replicated those previously found with pre/post data. The recovery trajectory was found to be linear, supporting our initial model (see Figure 2).

For all models, there was a significant interaction between the rate of change during the treatment period and baseline measurement of each dependent variable (e.g., pretreatment severity, PSWQ), such that higher baseline levels resulted in steeper recovery slopes. There were no significant differences between clients at posttreatment, given baseline measures. Nested model comparison using ANOVA demonstrated significantly improved fit when the interaction term was included (all Chi-sq values $p<.05$ ).

\section{Effect Sizes}

We also calculated the posttreatment and follow-up effect sizes for CBT + I/EP treatment by subtracting the pretreatment score from the posttreatment score and dividing this by the pretreatment $S D$. Similarly, we calculated the effect size at 1-year follow-up by subtracting the pretreatment score from the follow-up score and dividing it by the pretreatment $S D$. This was done for the three most commonly used measures of GAD outcome (assessor severity rating, Hamilton Anxiety Scale, and State Trait Anxiety Inventory) and averaged across the three measures at each time point. Table 2 presents CBT + I/EP effect size data. For the sake of providing some measure of comparison, albeit tentative, with the efficacy of CBT. Table 2 also includes average effect sizes from a meta-analysis by Borkovec and Ruscio (2001) of the extant CBT literature 


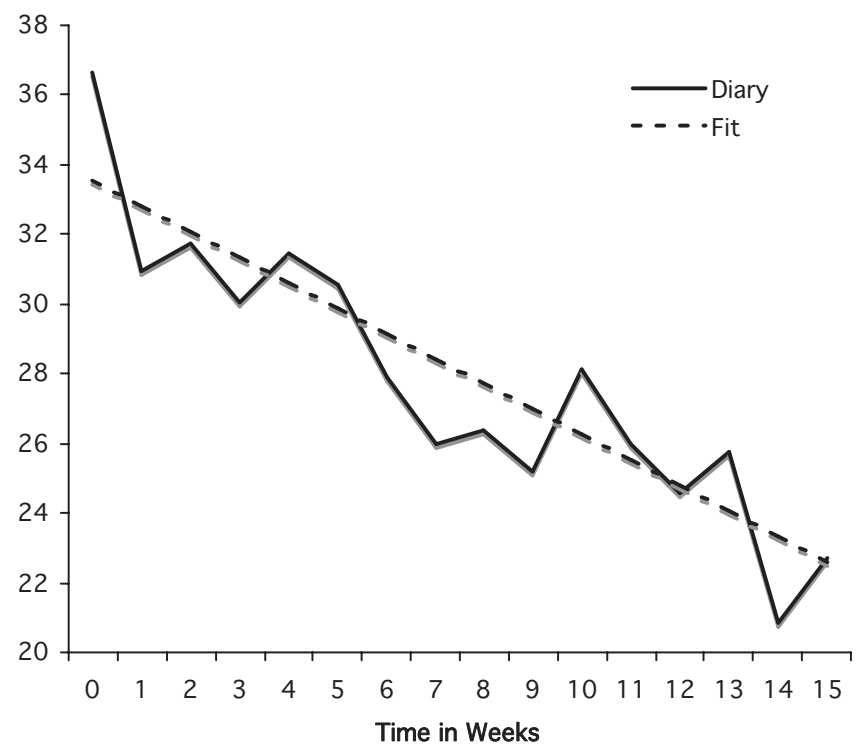

FIGURE 2. Actual and Modeled (Fit) Weekly Diary Scores During the Therapy Period.

and effect size data from Borkovec and colleagues' (2002), a pure trial of CBT. As demonstrated in this table, CBT/IEP has the highest effect sizes among these comparisons.

\section{Clinically Significant Change}

We calculated indices of clinically significant change in several ways. Similar to Borkovec and Costello (1993) responder status was defined by $20 \%$ change from pretherapy on at least four of the six primary outcome measures. Figure 3 shows the percentage of clients at each time point

TABLE 2. Average Within-Group Effect Sizes at PostTherapy and Follow-Up for Commonly Used Measures of Anxiety (Assessor Severity Rating, Hamilton Anxiety, STAI-Trait)

\begin{tabular}{|c|c|c|c|}
\hline & $\begin{array}{l}\text { Therapy } \\
\text { condition }\end{array}$ & Post-therapy & Follow-up \\
\hline \multicolumn{3}{|l|}{$\begin{array}{c}\text { Extant GAD outcome } \\
\text { studies }(N=11)\end{array}$} & 2.44 \\
\hline \multirow[t]{3}{*}{ Borkovec et al. 2002} & CT: & 2.94 & 2.48 \\
\hline & SCD: & 2.38 & 2.43 \\
\hline & CBT: & 2.80 & 2.43 \\
\hline \multirow[t]{2}{*}{ Current trial } & CBT/IEP: & 3.15 & 2.97 \\
\hline & & $N=18$ & $N=17$ \\
\hline
\end{tabular}

Note. $\mathrm{CT}=$ cognitive therapy; $\mathrm{SCD}=$ self-control coping desensitization; $\mathrm{CBT}=$ combined $\mathrm{CT}$ plus SCD; $\mathrm{CBT} / \mathrm{IEP}=$ cognitive behavioral therapy plus interpersonal and emotional processing. that met this criterion comparing the 18 clients in $\mathrm{CBT}+\mathrm{I} / \mathrm{EP}$ to the three clients who received CBT + SL. We also examined the impact of our therapy on clinically significant change in the IIP-C. We defined low endstate functioning on this measure as scoring at least $1 \mathrm{SD}$ above the mean on at least one of the eight IIP-C subscales. As demonstrated in Figure 4, whereas $95 \%$ of those receiving CBT + I/EP were considered low functioning interpersonally prior to therapy, only $55.6 \%$ of these clients remained low functioning at posttreatment. A McNemar's repeated measures chi-square test was used to examine changes in frequency of low endstate functioning on the IIP across time. There was a significant decline from pre- to posttreatment $(N=18$, ex act $p=.008), 6$-month follow-up $(N=16$, exact $p=.008)$ and 1-year assessment $(N=17$, exact $p=.008)$. There was no significant change when we examined the subset of clients from our previous CBT trial who received the IIP-C at pre- and postassessment $(N=35)$. Percentage of clients who met responder status on the IIP-C (at least a 20\% change on at least six of the eight IIP-C subscales) is depicted in Figure 5. Whereas the percentage of clients who met responder status in CBT $+\mathrm{I} / \mathrm{EP}$ increased during the follow-up period (as would be expected by interpersonal therapy theory (Wachtel, 1977)), this percentage decreased in the group of three clients in CBT + SL. 


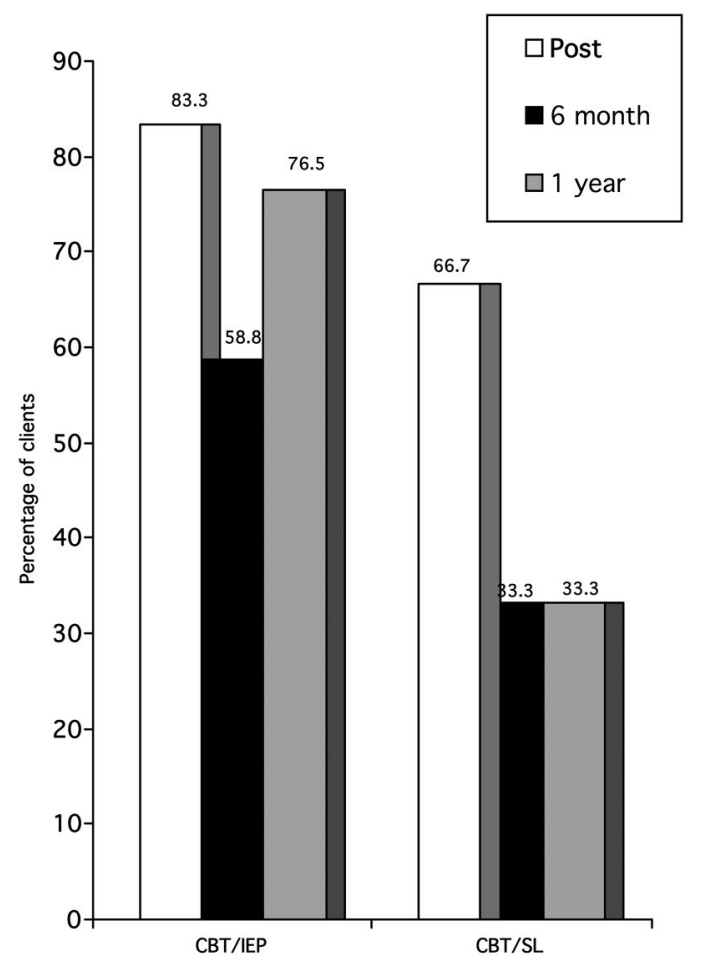

FIGURE 3. Comparison of cognitive-behavioral therapy plus interpersonal emotional processing therapy (CBT/IEP) to cognitive-behavioral therapy plus supportive listening (CBT/ SL). Percentage of clients who met responder status on at least four of six primary GAD outcome measures at each time point.

\section{Discussion}

The aim of the present study was to provide preliminary evidence for the feasibility and promising efficacy of a new integrative treatment for GAD that was specifically designed as an attempt to improve the efficacy of CBT, the only psychosocial intervention currently meeting criteria for the empirically supported treatment of GAD. Because the development and validation of CBT for GAD has been largely associated with the work of the third author, the current study should be viewed as a new step in a 20-year research program aimed at better understanding and treating one of the most prevalent and costly clinical disorders in our society. This new direction in our clinical and research efforts was guided by a substantial and cohesive amount of basic and applied research. Taken together, psychopathology research and studies on predictors of change (pretreatment, process, and posttreatment) have suggested that although important interper- sonal and emotional issues are involved in the etiology and/or maintenance of GAD, such issues do not appear to be adequately or fully addressed in current CBT protocols. Our efforts are also guided by the ultimate aim of empirical science, that is, the search for cause-and-effect relationships (Borkovec \& Castonguay, 1998). In addition to trying to improve CBT by adding techniques to facilitate interpersonal and emotional change, it is also important, for scientific reasons, to demonstrate that if such improvement takes place, it is most likely caused by the techniques added to the CBT protocol. Conjointly, these goals dictated the use of an additive design, which (assuming the demonstration of feasibility and promising efficacy) would provide the foundation for a later between-groups comparison study.

The current study provides support for the feasibility of combining, in a distinct and sequential manner, CBT and I/EP interventions. Adherence ratings demonstrated that therapists were able to apply techniques prescribed by these different forms of treatment, without major protocol breaches. In addition, qualitative ratings, conducted by an expert in the same protocols, indicated that the treatment could be implemented in

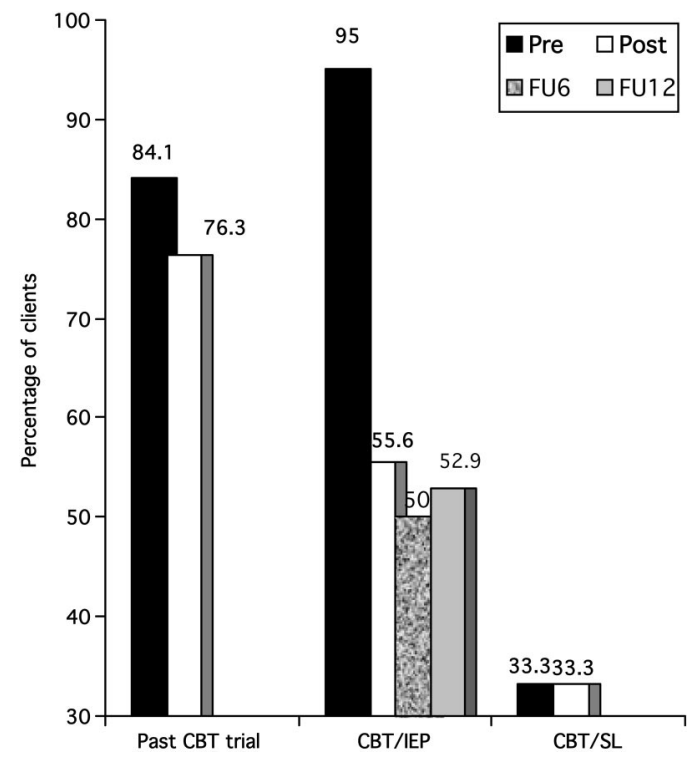

FIGURE 4. Comparison of clients from current trial to past CBT trial: percentage of clients who scored at least $1 S D$ above the mean of a normative sample on at least 1 Inventory of Interpersonal Problems scale at each time point. CBT/ IEP $=$ cognitive-behavioral therapy plus interpersonal emotional processing therapy; CBT/SL = cognitive-behavioral therapy plus supportive listening. 


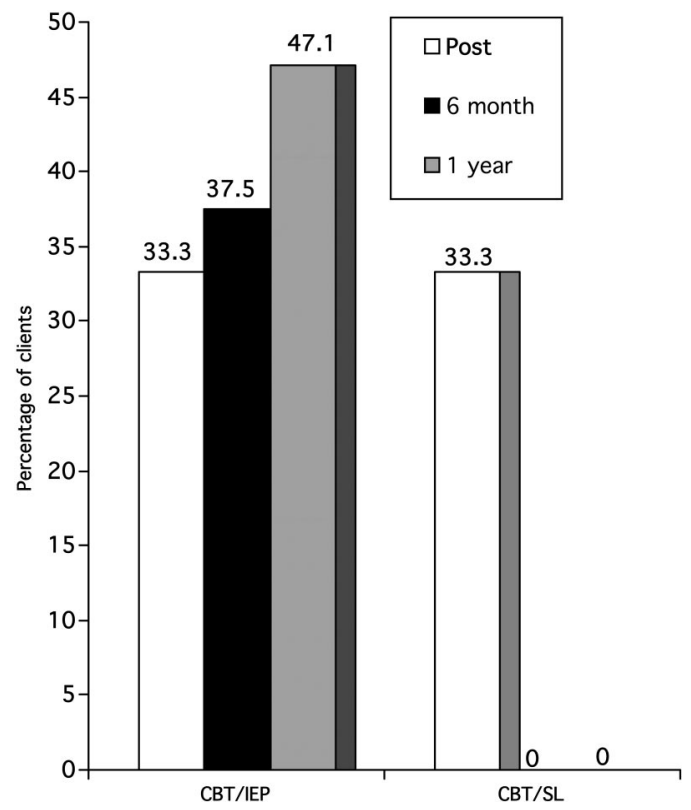

FIGURE 5. Comparison of cognitive-behavioral therapy plus interpersonal emotional processing therapy (CBT/IEP) to cognitive-behavioral therapy plus supportive listening (CBT/ SL). Percentage of clients who met criteria for clinically significant change on at least six of eight IIP scales at each time point

a competent manner. Moreover, anecdotal reports of both therapists and clients (as well as weekly therapy tape observations by the first three authors) suggested that the shift from one type of treatment to another within the same 2-hr sessions was not experienced as problematic or confusing for either clients or therapists. In fact, clients reported that the distinct interventions used in the different segments of therapy were complementary and had a synergic impact on many dimensions of their functioning.

The outcome findings of this open trial were also promising. Data indicated that participants' GAD symptoms decreased from pre to post therapy on all primary outcome measures and that this decrease was maintained during the 1-year follow-up period. Tests for random variation in the slope were not significant, suggesting that people did not differ significantly from the aggregate slope. The pre-to-post change pattern was replicated when we examined weekly daily diary data. In addition, analysis of the diary data demonstrated that the decrease during treatment was linear, as there were no nonlinear trends in these data. We also found that baseline assessor sever- ity ratings of GAD were significant as both a fixed and random effect, suggesting that most people ended up with the same level of improved GAD symptoms after treatment regardless of whether their severity level was high or low at the beginning of treatment. Thus, those with higher levels of GAD severity at pretreatment showed steeper levels of improvement than did those with lower levels of GAD severity at pretreatment.

Data from this open trial also suggested that most participants demonstrated clinically significant change on a number of indices with $76.5 \%$ of participants demonstrating responder status on primary GAD outcome measures at the 1-year follow-up point, as well as a significant decrease in the percentage of participants who had remaining interpersonal problems (i.e., scoring at least 1 $S D$ above the mean for a nonpsychiatric sample on at least one IIP-C subscale), a particular target of our integrative intervention. We also found that participants demonstrated continued improvement on interpersonal problems from posttreatment to follow-up. This is consistent with arguments by interpersonally oriented therapists that addressing interpersonal issues, especially as they emerge in therapy, is likely to have an impact on clients' interpersonal problems outside therapy (Henry \& Strupp, 1994). In addition, our results are consistent with the position held by interpersonal therapists that facilitating corrective interpersonal experiences during therapy (increasing awareness of relational needs, identifying past and current maladaptive relationship patterns, and testing new ways of relating to others) should also lead to incremental change after therapy (Wachtel, 1977). As clients continue to interact better with others, to learn more about their needs, and to achieve more satisfaction of these needs, they are likely to have a more gratifying and less problematic experience of life.

Several limitations of this study should be mentioned. We had a small sample size, of mostly Caucasian individuals, and a very limited control comparison with only three participants, which reduced our power to conduct any legitimate comparisons. In addition, our choice to separate the CBT and I/EP segments across two separate hours was for scientific purposes (additive design), which makes the protocol less likely to generalize to the real world of the practicing clinician. Nonetheless, this preliminary study encourages the use of a larger, between-groups design as a way to assess whether adding I/EP to 
CBT can increment therapeutic efficacy with GAD. Future studies may also test the feasibility of integrating in a more seamless manner techniques from different orientations that were included in the current proposal rather than keeping them separate. Such effectiveness studies, however, should not be conducted until issues of internal validity, and cause-effect relationships, are fully addressed.

\section{References}

Alden, L. E., \& Phillips, N. (1990). An interpersonal analysis of social anxiety and depression. Cognitive Therapy and Research, 14, 499-512.

Alden, L. E., Wiggins, J. S., \& Pincus, A. L. (1990). Construction of circumplex scales for the Inventory of Interpersonal Problems. Journal of Personality Assessment, 55, 521-536.

Bartholomew, K., \& Horowitz, L. M. (1991). Attachment styles among young adults: A test of a fourcategory model. Journal of Personality and Social Psychology, 61, 226-244.

Beck, A. T., Emery, G., \& GreenberG, R. L. (1985). Anxiety disorders and phobias: A cognitive perspective. New York: Basic Books.

Behar, E. S., \& Borkovec, T. D. (2003). Psychotherapy outcome research, In J. A. Schinka \& W. F. Velicer (Eds.), Handbook of psychology: Research methods in psychology (Vol. 2, pp. 213-240). New York, NY: John Wiley \& Sons, Inc.

BEn-Noun, L. (1998). Generalized anxiety disorder in dysfunctional families. Journal of Behavior Therapy and Experimental Psychiatry, 29, 115-122.

Bernstein, D. A., \& Borkovec, T. D. (1973). Progressive relaxation training: A manual for the helping professions. Champaign, IL: Research Press.

Blagys, M. D., \& Hilsenroth, M. J. (2000). Distinctive feature of short-term psychodynamic-interpersonal psychotherapy: A review of the comparative psychotherapy process literature. Clinical Psychology: Science and Practice, 7, 167-188.

Borkovec, T. D., Abel, J. L., \& Newman, H. (1995). Effects of psychotherapy on comorbid conditions in generalized anxiety disorder. Journal of Consulting and Clinical Psychology, 63, 479-483.

Borkovec, T. D., \& Castonguay, L. G. (1998). What is the scientific meaning of empirically supported therapy? Journal of Consulting and Clinical Psychology, 66, $136-142$.

Borkovec, T. D., \& Costello, E. (1993). Efficacy of applied relaxation and cognitive-behavioral therapy in the treatment of generalized anxiety disorder. Journal of Consulting and Clinical Psychology, 61, 611-619.

Borkovec, T. D., \& Hu, S. (1990). The effect of worry on cardiovascular response to phobic imagery. Behaviour Research and Therapy, 28, 69-73.

Borkovec, T. D., \& InZ, J. (1990). The nature of worry in generalized anxiety disorder: A predominance of thought activity. Behavior Research and Therapy, 28, 153-158.

Borkovec, T. D., \& NewMan, M. G. (1998). Worry and generalized anxiety disorder. In A. S. Bellack \& M. Hersen (Series Eds.), \& P. Salkovskis (Vol. Ed.), Comprehensive clinical psychology: Vol. 6. Adults: Clinical formulation and treatment. (pp. 439-459). Oxford, United Kingdom: Pergamon Press.

Borkovec, T. D., Newman, M. G., Pincus, A. L., \& LyTLE, R. (2002). A component analysis of cognitivebehavioral therapy for generalized anxiety disorder and the role of interpersonal problems. Journal of Consulting and Clinical Psychology, 70, 288-298.

Borkovec, T. D., Robinson, E., Pruzinsky, T., \& DePree, J. A. (1983). Preliminary exploration of worry: Some characteristics and processes. Behaviour Research and Therapy, 21, 9-16.

Borkovec, T. D., \& Roemer, L. (1995). Perceived functions of worry among generalized anxiety disorder subjects: Distraction from more emotionally distressing topics? Journal of Behavior Therapy and Experimental Psychiatry, 26, 25-30.

Borkovec, T. D., \& Ruscio, A. M. (2001). Psychotherapy for generalized anxiety disorder. Journal of Clinical Psychiatry, 62, 37-45.

Brawman-Mintzer, O., Lydiard, R. B., Emmanuel, N., Payeur, R., Johnson, M., Roberts, J., et Al. (1993). Psychiatric comorbidity in patients with GAD. American Journal of Psychiatry, 150, 1216-1218.

Breitholtz, E., Johansson, B., \& Ost, L. G. (1995). Cognitions in generalized anxiety disorder and panic disorder patients: A prospective approach. Behaviour Research and Therapy, 37, 533-544.

Brown, T. A., Antony, M. M., \& Barlow, D. H. (1992). Psychometric properties of the Penn State Worry Questionnaire in a clinical anxiety disorders sample. Behaviour Research and Therapy, 30, 33-37.

Brown, T. A., Barlow, D. H., \& Liebowitz, M. R. (1994). The empirical basis of generalized anxiety disorder. American Journal of Psychiatry, 151, 1272-1280.

Brown, T. A., Campbell, L. A., Lehman, C. L., Grisham, J. R., \& MAnCILL, R. B. (2001). Current and lifetime comorbidity of the DSM-IV anxiety and mood disorders in a large clinical sample. Journal of Abnormal Psychology, 110, 585-599.

Brown, T. A., Di Nardo, P. A., \& Barlow, D. H. (1994). Anxiety disorders interview schedule for DSM$I V$. New York: Oxford University Press.

Bruss, G. S., Gruenberg, A. M., Goldstein, R. D., \& BARber, J. P. (1994). Hamilton Anxiety Rating Scale Interview Guide: Joint interview and test-retest methods for interrater reliability. Psychiatry Research, 53, 191-202.

Castonguay, L. G., Goldfried, M. R., Wiser, S., Raue, P. J., \& Hayes, A. M. (1996). Predicting the effect of cognitive therapy for depression: A study of unique and common factors. Journal of Consulting and Clinical Psychology, 64, 497-504.

ERICKSON, T. M., \& NeWMAN, M. G. (2007). Interpersonal and emotional processes in generalized anxiety disorder analogues during social interaction tasks. $B e$ havior Therapy, 38, 364-377.

First, M. B., Spitzer, R. L., Gibbon, M., Williams, J. B. W., \& Benjamin, L. (1994). Structured clinical interview for DSM-IV Axis II personality disorders (SCID II), version 2.0. New York: Biometrics Research Department. 
FoA, E. B., \& KozAK, M. J. (1986). Emotional processing of fear: Exposure to corrective information. Psychological Bulletin, 99, 20-35.

Gasperini, M., Battaglia, M., Diaferia, G., \& BELlOdI, L. (1990). Personality features related to generalized anxiety disorder. Comprehensive Psychiatry, 31, 363-368.

HAMiLTON, M. (1959). The assessment of anxiety states by rating. British Journal of Medical Psychology, 32, $50-55$.

Heide, F. J., \& Borkovec, T. D. (1983). Relaxationinduced anxiety: Paradoxical anxiety enhancement due to relaxation training. Journal of Consulting and Clinical Psychology, 51, 171-182.

Henry, W. P., \& Strupp, H. H. (1994). The therapeutic alliance as interpersonal process. In A. O. Horvath \& L. S. Greenberg (Eds.), The working alliance: Theory, research, and practice (pp. 51-84). New York: Wiley.

Kobak, K. A., Reynolds, W. M., \& Greist, J. H. (1993). Development and validation of a computer-administered version of the Hamilton Rating Scale. Psychological Assessment, 5, 487-492.

Llera, S. J., \& Newman, M. G. (2007, November). Emotional avoidance in GAD: The moderating effects of worry on both subjective and physiological emotional responding. Paper presented at the 41st Annual meeting of the Association for the Advancement of Behavioral and Cognitive Therapies, Philadelphia.

Maier, W., Buller, R., Philipp, M., \& Heuser, I. (1988). The Hamilton Anxiety Scale: Reliability, validity and sensitivity to change in anxiety and depressive disorders. Journal of Affective Disorders, 14, 61-68.

Mennin, D. S., Heimberg, R. G., Turk, C. L., \& Fresco, D. M. (2005). Preliminary evidence for an emotion dysregulation model of generalized anxiety disorder. Behaviour Research and Therapy, 43, 1281-1310.

Meyer, T. J., Miller, M. L., Metzger, R. L., \& Borkovec, T. D. (1990). Development and validation of the Penn State Worry Questionnaire. Behaviour Research and Therapy, 28, 487-495.

Molina, S., \& Borkovec, T. D. (1994). The Penn State Worry Questionnaire: Psychometric properties and associated characteristics. In G. C. L. Davey \& F. Tallis (Eds.), Worrying: Perspectives on theory, assessment and treatment (pp. 265-283). Oxford, United Kingdom: Wiley.

Newman, M. G. (2000). Recommendations for a costoffset model of psychotherapy allocation using generalized anxiety disorder as an example. Journal of Consulting and Clinical Psychology, 68, 549-555.

Newman, M. G., Castonguay, L. G., Borkovec, T. D., \& Molnar, C. (2004). Integrative psychotherapy. In R. G. Heimberg, C. L. Turk \& D. S. Mennin (Eds.), Generalized anxiety disorder: Advances in research and practice. (pp. 320-350). New York: Guilford Press.

Newman, M. G., Przeworski, A., Fisher, A. J., \&
Borkovec, T. D. (2007). Diagnostic comorbidity in a randomized controlled trial of adults with generalized anxiety disorder: Impact of comorbidity on psychotherapy outcome and impact of psychotherapy on comorbid diagnoses. Manuscript submitted for publication.

Newman, M. G., Zuellig, A. R., Kachin, K. E., Constantino, M. J., Przeworski, A., Erickson, T., et al. (2002). Preliminary reliability and validity of the Generalized Anxiety Disorder Questionnaire-IV: A revised self-report diagnostic measure of generalized anxiety disorder. Behavior Therapy, 33, 215-233.

Pincus, A. L., \& Borkovec, T. D. (1994, June). Interpersonal problems in generalized anxiety disorder: Preliminary clustering of patients' interpersonal dysfunction. Paper presented at the Annual Meeting of the American Psychological Society, New York.

Roemer, L., Molina, S., \& Borkovec, T. D. (1997). An investigation of worry content among generally anxious individuals. Journal of Nervous and Mental Disease, 185, 314-319.

SAFran, J. D., \& SEgAL, Z. V. (1990). Interpersonal process in cognitive therapy. New York: Basic Books.

Snaith, R. P., Harrop, F. M., Newby, D. A., \& Teale, C. (1986). Grade scores of the Montgomery-Ösberg Depression and the Clinical Anxiety Scales. British Journal of Psychiatry, 148, 599-601.

Spielberger, C. D., Gorsuch, R. L., Lushene, R., VAGG, P. R., \& JACOBS, G. A. (1983). Manual for the State-Trait Anxiety Inventory STAI (Form Y). Palo Alto, CA: Mind Garden.

Tucker, D. M., \& Newman, J. P. (1981). Verbal versus imaginal cognitive strategies in the inhibition of emotional arousal. Cognitive Therapy and Research, 5, 197-202.

Turk, C. L., Heimberg, R. G., Luterek, J. A., Mennin, D. S., \& Fresco, D. M. (2005). Emotion dysregulation in generalized anxiety disorder: A comparison with social anxiety disorder. Cognitive Therapy and Research, 5, 89-106.

Wachtel, P. L. (1977). Psychoanalysis and behavior therapy: Toward an integration. New York: Basic Books.

Whisman, M. A., Sheldon, C. T., \& Goering, P. (2000). Psychiatric disorders and dissatisfaction with social relationships: Does type of relationship matter? Journal of Abnormal Psychology, 109, 803-808.

YAMASAKI, A. S., Behar, E., \& Ray, W. J. (2002, November). Is there a failure to process general emotionality, regardless of valence in generalized anxiety disorder? Paper presented at the 36th Annual Meeting of the Association for Advancement of Behavior Therapy, Reno, NV.

Young, J. E., \& Beck, A. T. (1980). The development of the cognitive therapy scale. Philadelphia, PA: Center for Cognitive Therapy. 